\title{
The Three Dimensional Structure of CMEs from LASCO Polarization Measurements
}

Kenneth Dere ${ }^{1}$, and Dennis Wang ${ }^{2,3}$

${ }^{1}$ George Mason University, USA email: kdere@gmu.edu

${ }^{2}$ Interferometrics, Inc., email: dennis.wang@nrl.navy.mil

\begin{abstract}
The degree of polarization of Compton-scattered photospheric light observed in a coronagraph is dependent on the distance of the scattering electrons from the plane of the sky. Measurements of the polarization of light scattered by CME structures have been observed by LASCO C2. We have reduced and analyzed a month long sequence of such measurements which were taken at a cadence of 1 hour. The CME brightness has been distributed throughout a 3 dimensional cube and visualized at a variety of angles. Several CMEs are found to have considerable fine-structure consistent with expanding loop arcades. The analysis is subject to a variety of assumptions such as a lack of knowledge of whether a source is before or behind the plane of the sky. Nevertheless, the results obtained to date are intriguing.
\end{abstract}

Keywords. polarization, Sun: coronal mass ejections (CMEs)

\section{References}

Dere, K. P., Wang, D., \& Howard, R. A. 2005, ApJL, 620, 119 